Table 1. Atomic positional parameters and $B_{\mathrm{eq}}$ values for (1), with e.s.d.'s

$$
\begin{gathered}
B_{\text {eq }}=(4 / 3)\left[a^{2} B_{11}+b^{2} B_{22}+c^{2} B_{33}+(2 a b \cos \gamma) B_{12}+(2 a c \cos \beta) B_{13}\right. \\
\left.+(2 b c \cos \alpha) B_{23}\right] .
\end{gathered}
$$

$\begin{array}{lcccl} & x & y & z & B_{\mathrm{eq}}\left(\AA^{2}\right) \\ \mathrm{Br} & 0.28344(7) & 0.11981(3) & 0.07820(6) & 4.15(1) \\ \mathrm{O} & -0.2308(5) & 0.1133(2) & -0.6789(4) & 4.58(9) \\ \mathrm{N} 1 & -0.3496(5) & -0.0173(2) & -0.6189(5) & 2.96(9) \\ \mathrm{N} 2 & -0.4606(5) & -0.1322(2) & -0.5533(5) & 3.5(1) \\ \mathrm{C} 1 & 0.0105(6) & 0.0545(3) & -0.2247(5) & 3.1(1) \\ \mathrm{C} 2 & 0.1231(6) & 0.1160(3) & -0.1620(6) & 3.1(1) \\ \mathrm{C} 3 & 0.1201(6) & 0.1751(3) & -0.2688(7) & 3.7(1) \\ \mathrm{C} 4 & 0.0031(6) & 0.1739(3) & -0.4422(6) & 3.7(1) \\ \mathrm{C} 5 & -0.1156(6) & 0.1123(3) & -0.5095(6) & 3.2(1) \\ \mathrm{C} 6 & -0.1100(6) & 0.0524(3) & -0.3981(5) & 2.7(1) \\ \mathrm{C} 7 & -0.2319(6) & -0.0131(3) & -0.4610(5) & 3.0(1) \\ \mathrm{C} 8 & -0.4661(5) & -0.0817(3) & -0.6724(5) & 2.9(1) \\ \mathrm{C} 9 & -0.5769(6) & -0.0885(3) & -0.8458(6) & 3.4(1) \\ \mathrm{C} 10 & -0.6871(6) & -0.1520(3) & -0.9028(6) & 3.7(1) \\ \mathrm{C} 11 & -0.6829(6) & -0.2049(3) & -0.7830(7) & 3.6(1) \\ \mathrm{C} 12 & -0.5697(7) & -0.1925(3) & -0.6117(6) & 3.9(1) \\ \mathrm{H}^{\prime} \mathrm{O} & -0.276(5) & 0.076(3) & -0.697(5) & 4 *\end{array}$

* Refined isotropically.

Table 2. Selected bond distances $(\AA)$ and bond angles $\left({ }^{\circ}\right)$ with e.s.d.'s in parentheses

\begin{tabular}{|c|c|c|c|}
\hline $\mathrm{C} 5-\mathrm{O}-\mathrm{H}^{\prime} \mathrm{O}$ & $106(3)$ & $\mathrm{Cl}-\mathrm{C} 6-\mathrm{C} 7$ & $118 \cdot 7$ \\
\hline $\mathrm{C} 7-\mathrm{NI}-\mathrm{C} 8$ & $119 \cdot 2(4)$ & $\mathrm{C} 5-\mathrm{C} 6-\mathrm{C} 7$ & $121 \cdot 2$ \\
\hline $\mathrm{C} 8-\mathrm{N} 2-\mathrm{C} 12$ & $116 \cdot 1(4)$ & $\mathrm{N} 1-\mathrm{C} 7-\mathrm{C} 6$ & $121 \cdot 6$ \\
\hline $\mathrm{C} 2-\mathrm{C} 1-\mathrm{C} 6$ & $119 \cdot 8(4)$ & $\mathrm{N} 1-\mathrm{C} 8-\mathrm{N} 2$ & $118 \cdot 8$ \\
\hline $\mathrm{Cl}-\mathrm{C} 2-\mathrm{C} 3$ & $121 \cdot 0(4)$ & $\mathrm{N} 1-\mathrm{C} 8-\mathrm{C} 9$ & $117 \cdot 7$ \\
\hline $\mathrm{C} 2-\mathrm{C} 3-\mathrm{C} 4$ & $120 \cdot 3(4)$ & $\mathrm{N} 2-\mathrm{C} 8-\mathrm{C} 9$ & 123.5 \\
\hline $\mathrm{C} 3-\mathrm{C} 4-\mathrm{C} 5$ & $119.9(5)$ & $\mathrm{C} 8-\mathrm{C} 9-\mathrm{C} 10$ & $119 \cdot 0$ \\
\hline $\mathrm{O}-\mathrm{C} 5-\mathrm{C} 4$ & $118.6(4)$ & $\mathrm{C} 9-\mathrm{Cl}-\mathrm{Cl}$ & 118.5 \\
\hline $\mathrm{O}-\mathrm{C} 5-\mathrm{C} 6$ & $122.6(4)$ & $\mathrm{C} 10-\mathrm{C} 11-\mathrm{C} 12$ & 118.2 \\
\hline $\mathrm{C} 4-\mathrm{C} 5-\mathrm{C} 6$ & $118 \cdot 8(4)$ & $\mathrm{N} 2-\mathrm{C} 12-\mathrm{C} 11$ & $124 \cdot 6$ \\
\hline $\mathrm{Cl}-\mathrm{C} 6-\mathrm{C} 5$ & $120 \cdot 1(4)$ & & \\
\hline
\end{tabular}

$\begin{array}{llll}\mathrm{O}-\mathrm{C} 5 & 1.346(5) & \mathrm{C} 3-\mathrm{C} 4 & 1.376(6) \\ \mathrm{O}-\mathrm{H} \mathrm{O} & 0.73(5) & \mathrm{C} 4-\mathrm{C} 5 & 1.404(7) \\ \mathrm{N} 1-\mathrm{C} 7 & 1.281(5) & \mathrm{C} 5-\mathrm{C} 6 & 1.399(7) \\ \mathrm{N} 1-\mathrm{C} 8 & 1.424(6) & \mathrm{C} 6-\mathrm{C} 7 & 1.466(6) \\ \mathrm{N} 2-\mathrm{C} 8 & 1.329(6) & \mathrm{C} 8-\mathrm{C} 9 & 1.369(6) \\ \mathrm{N} 2-\mathrm{C} 12 & 1.337(6) & \mathrm{C} 9-\mathrm{C} 10 & 1.386(7) \\ \mathrm{C} 1-\mathrm{C} 2 & 1.371(6) & \mathrm{C} 10-\mathrm{H} 10 & 0.98(4) \\ \mathrm{Cl}-\mathrm{C} 6 & 1.384(5) & \mathrm{C} 11-\mathrm{C} 12 & 1.372(6) \\ \mathrm{C} 2-\mathrm{C} 3 & 1.369(7) & & \end{array}$

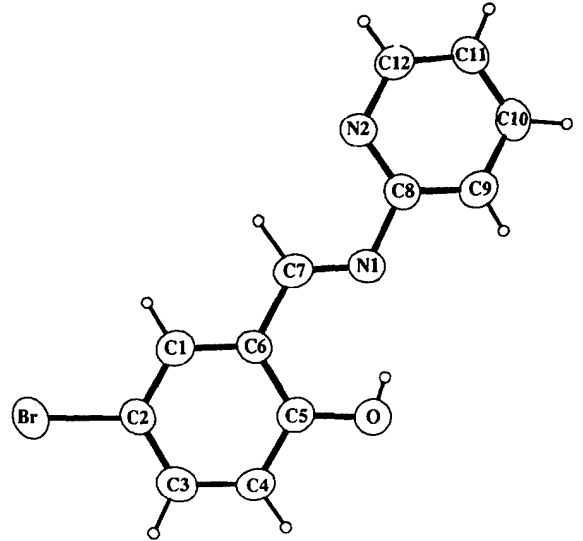

Fig. 1. Molecular structure of the title compound, with atomnumbering scheme.

1984). The 5-bromo derivative was synthetized because 2-(2-pyridyliminomethyl)phenol (salapy) did not give good crystals for X-ray diffraction studies.

\section{References}

Escobar, C. \& Garland, M. T. (1983). Acta Cryst. C39, 1463-1465.

Escobar, C. \& Garland, M. T. (1984). Acta Cryst. C40, 889-891.

Frenz, B. A. (1983). Enraf-Nonius Structure Determination Package; SDP Users Guide. Version of 6 January 1983. EnrafNonius, Delft, The Netherlands.

Main, P., Fiske, S. J., Hull, S. E., Lessinger, L., Germain, G., DeClercQ, J.-P. \& Woolfson, M. M. (1980). MULTAN80. A System of Computer Programs for the Automatic Solution of Crystal Structures from X-ray Diffraction Data. Univs. of York, England, and Louvain, Belgium.

Stewart, J. M., Machin, P. A., Dickinson, C. W., Ammon, H. L., HeCK, H. \& FlaCK, H. (1976). The XRAY76 system. Tech. Rep. TR-446. Computer Science Center, Univ. of Maryland, College Park, Maryland, USA.

Stout, G. H. \& JenSEN, L. H. (1968). In X-ray Structure Determination. New York: Macmillan.

Acta Cryst. (1991). C47, 1117-1120

\title{
Structure of a Fully Protected Seco-erythronolide B Acid Derivative
}

\author{
By V. M. Lynch, W.-C. LeE, S. F. Martin and B. E. Davis \\ Department of Chemistry and Biochemistry, University of Texas at Austin, Austin, TX 78712, USA
}

(Received 15 August 1990; accepted 25 October 1990)

\begin{abstract}
S, 4 R, 5 S, 6 R, 7 R, 9 R, 10 S, 11 S, 12 S, 13 S$,$14 R$ )-14-Benzyloxymethoxy-10,12-O-carbonyl-4- $N$ imidazolylcarbonyl-6,7-O-isopropylidene-3,5,7,9,11,13-hexamethylhexadec-1-ene-4,6,7,10,12,14-hexol,
\end{abstract}

$\mathrm{C}_{38} \mathrm{H}_{56} \mathrm{~N}_{2} \mathrm{O}_{9}, \quad M_{r}=684.87$, monoclinic, $P 2_{1}, \quad a=$ 8.7302 (12), $b=15.597$ (2), $c=14.463(2) \AA, \quad \beta=$ $104.797(10)^{\circ}, V=1904 \cdot 1$ (4) $\AA^{3}, Z=2, D_{x}(198 \mathrm{~K})$ $=1.19 \mathrm{~g} \mathrm{~cm}^{-3}, \mu=0.7893 \mathrm{~cm}^{-1}$, Mo $K \alpha$ radiation, (C) 1991 International Union of Crystallography 
$\lambda=0.7107 \AA, F(000)=740, T=198 \mathrm{~K}, R=0.0301$ for 3141 reflections $\left[F_{o} \geq 4 \sigma\left(F_{o}\right)\right]$. The crystal structure was undertaken to determine the stereochemistry of the title compound. The molecule is folded on itself in such a way that the portion of the molecule from the phenyl ring at $\mathrm{C} 44$ and extending to C28 forms nearly a single turn of a right-handed screw. Large deviations from ideality for several $\mathrm{Cs} p^{3}-\mathrm{C} s p^{3}$ bond angles are observed that are presumably due to intramolecular steric effects. The largest deviations are: $\mathrm{C} 3-\mathrm{C} 4-\mathrm{C} 5 \quad 117.8(2)$, C5-C6-C7 121.2(2), C7-C8-C9 115.3 (2), C9$\mathrm{C} 10-\mathrm{C} 11 \quad 116 \cdot 2(2), \quad \mathrm{C} 11-\mathrm{C} 12-\mathrm{C} 13 \quad 117 \cdot 2(2)$, $\mathrm{C} 14-\mathrm{C} 15-\mathrm{C} 16115 \cdot 5(3)^{\circ}$.

Experimental. Compound (1) was synthesized in two steps by the asymmetric reduction of (2) (Martin, Pacofsky, Gist \& Lee, 1989) with tetramethylammonium triacetoxyborohydride $\left[\mathrm{Me}_{4} \mathrm{NBH}(\mathrm{OAc})_{3}\right]$ (Evans, Chapman \& Carreira, 1988) followed by refluxing with $1,1^{\prime}$-carbonyldiimidazole in benzene.

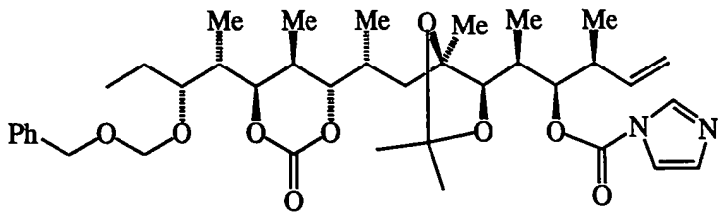

(1)

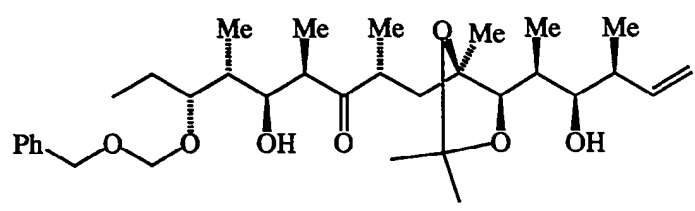

(2)

Full details of the synthetic procedure will be described in due course (Martin \& Lee, 1990). Crystals were obtained by slow evaporation of a hexanediethyl ether solution. The data crystal was a clear, colorless plate of approximate dimensions $0.23 \times$ $0.45 \times 0.52 \mathrm{~mm}$ that was cut from a larger crystal. The data were collected on a Nicolet $R 3$ diffractometer using a graphite monochromator and a Nicolet LT-2 low-temperature delivery system. Lattice parameters were obtained from the least-squares refinement of 40 reflections with $24.4<2 \theta<28.0^{\circ}$. The data were collected using the $\omega$-scan technique with a $2 \theta$ range $4 \cdot 0-50 \cdot 0^{\circ}$, with a $1 \cdot 2^{\circ} \omega$ scan at $6-12^{\circ} \min ^{-1}(h-10 \rightarrow 10, k 0 \rightarrow 18, l-17 \rightarrow 17)$. A total of 7035 reflections were collected of which 3520 were unique $\left(R_{\text {int }}=0.0143\right.$ for averaging symmetryequivalent reflections). Four reflections (143, 124, $1 \overline{4}$, 115 ) were remeasured every 196 reflections to
Table 1. Fractional coordinates and equivalent isotropic thermal parameters $\left(\AA^{2}\right)$ for the nonhydrogen atoms of (1)

For anisotropic atoms, the $U$ value is $U_{\text {eq }}$, calculated as $U_{\text {eq }}=$ $\frac{1}{3} \sum_{i} \sum_{j} U_{i j} a_{i}^{*} a_{j}^{*} \mathbf{A}_{i j}$ where $\mathbf{A}_{i j}$ is the dot product of the $i$ th and $j$ th direct-space unit-cell vectors.

\begin{tabular}{|c|c|c|c|c|}
\hline & $x$ & $y$ & $z$ & $U$ \\
\hline $\mathrm{Cl}$ & $0.7245(4)$ & $1.0995(2)$ & $0.6967(2)$ & $0.0622(12)$ \\
\hline $\mathrm{C} 2$ & $0.6189(3)$ & $1.0930(2)$ & $0.7462(2)$ & $0.0479(9)$ \\
\hline C3 & $0.6493(3)$ & $1.0665(2)$ & $0.8498(2)$ & $0.0399(8)$ \\
\hline $\mathrm{C4}$ & $0.5670(3)$ & $0.98045(15)$ & $0.85469(15)$ & $0.0311(7)$ \\
\hline $\mathrm{C} 5$ & $0.3869(3)$ & $0.97592(14)$ & $0.8122(2)$ & $0.0333(7)$ \\
\hline C6 & $0.3339(3)$ & $0.88264(14)$ & $0.81719(14)$ & $0.0316(7)$ \\
\hline$C 7$ & $0 \cdot 1801(2)$ & $0.84912(13)$ & $0.74952(14)$ & $0.0309(7)$ \\
\hline $\mathrm{C} 8$ & $0.2059(3)$ & $0.81927(15)$ & $0.65442(15)$ & $0.0309(7)$ \\
\hline C9 & $0.0598(2)$ & $0.77845(15)$ & $0.58457(14)$ & $0.0331(7)$ \\
\hline $\mathrm{ClO}$ & $0 \cdot 1078(3)$ & $0.69390(15)$ & $0 \cdot 5465(2)$ & $0.0370(7)$ \\
\hline $\mathrm{C} 11$ & $0 \cdot 1484(3)$ & $0.62101(14)$ & $0 \cdot 6177(2)$ & $0.0338(7)$ \\
\hline $\mathrm{Cl} 2$ & $-0.0044(2)$ & $0.58969(15)$ & $0.63804(15)$ & $0.0334(7)$ \\
\hline $\mathrm{Cl3}$ & $0.0075(3)$ & $0.51398(15)$ & $0.7064(3)$ & $0.0368(8)$ \\
\hline $\mathrm{Cl}$ & $-0 \cdot 1509(3)$ & $0.4957(2)$ & $0.7296(2)$ & $0.0450(9)$ \\
\hline $\mathrm{C} 15$ & $-0 \cdot 1563(4)$ & $0.4115(2)$ & $0.7820(2)$ & $0.0598(12)$ \\
\hline $\mathrm{C} 16$ & $-0.1338(4)$ & $0 \cdot 3322(2)$ & $0.7287(3)$ & $0.0671(13)$ \\
\hline $\mathrm{Cl7}$ & $0 \cdot 8248(3)$ & $1.0613(2)$ & 0.9009 (2) & $0.0503(9)$ \\
\hline 018 & $0.5960(2)$ & $0.95666(10)$ & $0.95660(10)$ & $0.0351(5)$ \\
\hline $\mathrm{Cl9}$ & $0.6586(3)$ & $0 \cdot 8802(2)$ & $0.98143(15)$ & $0.0364(7)$ \\
\hline $\mathrm{O} 20$ & $0 \cdot 7221$ (2) & $0.83355(12)$ & $0.93705(12)$ & $0.0518(6)$ \\
\hline $\mathrm{N} 21$ & $0 \cdot 6438(2)$ & $0.85876(13)$ & $1.07328(12)$ & $0.0388(6)$ \\
\hline $\mathrm{C} 22$ & $0.5445(3)$ & $0.8942(2)$ & l·1221 (2) & $0.0515(10)$ \\
\hline $\mathrm{N} 23$ & $0.5572(3)$ & $0.8567(2)$ & $1 \cdot 2042(2)$ & $0.0676(10)$ \\
\hline $\mathrm{C} 24$ & 0.6699 (4) & $0.7931(2)$ & $1 \cdot 2083(2)$ & $0.0665(12)$ \\
\hline $\mathrm{C} 25$ & $0.7235(3)$ & $0.7926(2)$ & $1 \cdot 1295(2)$ & $0.0541(10)$ \\
\hline $\mathrm{C} 26$ & $0 \cdot 2991$ (3) & $1.0416(2)$ & $0 \cdot 8585(2)$ & 0.0477 (9) \\
\hline $\mathrm{O} 27$ & $0.3095(2)$ & $0.86427(10)$ & $0.90943(10)$ & $0.0416(5)$ \\
\hline C28 & $0 \cdot 2346(3)$ & $0.7823(2)$ & $0.9014(2)$ & $0.0432(8)$ \\
\hline $\mathrm{O} 29$ & $0.1537(2)$ & $0.77310(11)$ & $0.80187(10)$ & $0.0387(5)$ \\
\hline C30 & $0 \cdot 1169(5)$ & $0.7827(3)$ & $0.9618(2)$ & $0.0689(13)$ \\
\hline C31 & $0.3594(4)$ & $0.7127(2)$ & $0.9293(2)$ & $0.0552(10)$ \\
\hline $\mathrm{C} 32$ & $0.0388(3)$ & $0.9086(2)$ & $0.7375(2)$ & $0.0452(9)$ \\
\hline C33 & $-0.0133(4)$ & $0 \cdot 8409(2)$ & $0.5039(2)$ & $0.0559(10)$ \\
\hline O34 & $-0.0186(2)$ & $0.66622(12)$ & $0.46321(10)$ & $0.0496(6)$ \\
\hline $\mathrm{C} 35$ & $-0.1194(3)$ & $0.6030(2)$ & $0.4659(2)$ & $0.0440(8)$ \\
\hline O36 & $-0.1129(2)$ & $0.56202(11)$ & $0.54783(11)$ & $0.0437(5)$ \\
\hline $\mathrm{O} 37$ & $-0.2161(2)$ & $0.58237(13)$ & $0.39407(12)$ & $0.0602(7)$ \\
\hline $\mathrm{C} 38$ & $0.2414(3)$ & $0.5508(2)$ & $0.5827(2)$ & $0.0496(10)$ \\
\hline C39 & $0 \cdot 1364(4)$ & $0.5313(2)$ & $0.7978(2)$ & $0.0524(10)$ \\
\hline 040 & $-0.1779(2)$ & $0.56568(13)$ & 0.78912 (11) & $0.0535(6)$ \\
\hline C41 & $-0.3380(4)$ & $0 \cdot 5866(3)$ & $0.7785(3)$ & 0.0670 (13) \\
\hline O42 & $-0.3977(2)$ & $0.63977(13)$ & $0.69905(14)$ & $0.0610(7)$ \\
\hline $\mathrm{C} 43$ & $-0.3335(3)$ & $0.7243(2)$ & $0.7103(2)$ & $0.0503(10)$ \\
\hline C44 & $-0.3847(2)$ & $0.7710(2)$ & $0.6170(2)$ & $0.0395(8)$ \\
\hline $\mathrm{C} 45$ & $-0.4103(3)$ & $0.7283(2)$ & $0 \cdot 5300(2)$ & $0.0488(10)$ \\
\hline C46 & $-0.4539(3)$ & 0.7735 (2) & $0.4449(2)$ & $0.0560(10)$ \\
\hline $\mathrm{C} 47$ & $-0.4731(3)$ & $0.8611(2)$ & $0.4448(2)$ & $0.0558(10)$ \\
\hline $\mathrm{C} 48$ & $-0.4496(3)$ & 0.9041 (2) & $0.5305(2)$ & $0.0509(10)$ \\
\hline C49 & $-0.4054(3)$ & $0.8594(2)$ & $0.6149(2)$ & $0.0434(9)$ \\
\hline
\end{tabular}

monitor instrument and crystal stability. A smoothed curve of the intensities of these check reflections was used to scale the data. The scaling factor ranged from $0.966-1.03$. The data were also corrected for $L p$ effects but not absorption. The data reduction and decay correction were applied using the Nicolet XRD SHELXTL-Plus software package (Sheldrick, 1988). Reflections having $F_{o}<4 \sigma\left(F_{o}\right)$ were considered unobserved (379 reflections). The structure was solved by direct methods (Sheldrick, 1988) and refined by full-matrix least squares (Sheldrick, 1976). In all, 666 parameters were refined in blocks of 325 and 342 parameters with the scale factor refined in each block. The non-H atoms were refined with anisotropic thermal parameters. All $\mathrm{H}$-atom positions were obtained from a $\Delta F$ map and refined with 
Table 2. Bond lengths $(\AA)$ and angles $\left(^{\circ}\right)$ for the non-hydrogen atoms of (1)

\begin{tabular}{|c|c|c|c|c|}
\hline 1 & 2 & 3 & $1-2$ & $1-2-3$ \\
\hline $\mathrm{C} 2$ & $\mathrm{Cl}$ & & $1 \cdot 308(5)$ & \\
\hline C3 & $\mathrm{C} 2$ & $\mathrm{Cl}$ & $1 \cdot 511(4)$ & $126 \cdot 4(3)$ \\
\hline $\mathrm{C4}$ & C3 & $\mathrm{Cl} 17$ & $1.532(3)$ & $110.9(2)$ \\
\hline C4 & C3 & $\mathrm{C} 2$ & & $108 \cdot 6(2)$ \\
\hline $\mathrm{Cl} 7$ & C3 & $\mathrm{C} 2$ & $1 \cdot 524(3)$ & $113 \cdot 3(2)$ \\
\hline C5 & $\mathrm{C} 4$ & 018 & $1 \cdot 536(3)$ & $106 \cdot 6(2)$ \\
\hline C5 & $\mathrm{CA}$ & C3 & & $117.8(2)$ \\
\hline 018 & C4 & C3 & $1.478(3)$ & $107 \cdot 6(2)$ \\
\hline C6 & $\mathrm{C} 5$ & $\mathrm{C} 26$ & $1.534(3)$ & $115.0(2)$ \\
\hline C6 & C5 & $\mathrm{C} 4$ & & $108 \cdot 3(2)$ \\
\hline $\mathrm{C} 26$ & C5 & $\mathrm{C} 4$ & $1 \cdot 532(4)$ & $111 \cdot 7(2)$ \\
\hline C7 & C6 & $\mathrm{O} 27$ & $1.537(3)$ & $102 \cdot 2(2)$ \\
\hline C7 & C6 & C5 & & $121 \cdot 2(2)$ \\
\hline $\mathrm{O} 27$ & C6 & $\mathrm{C} 5$ & $1.433(3)$ & $110 \cdot 6(2)$ \\
\hline $\mathrm{C} 8$ & C7 & $\mathrm{O} 29$ & $1.523(3)$ & $107.5(2)$ \\
\hline $\mathrm{C} 8$ & C7 & C32 & & $112.8(2)$ \\
\hline C8 & C7 & C6 & & $111.8(2)$ \\
\hline O29 & $\mathrm{C} 7$ & C 32 & $1.456(3)$ & $109.0(2)$ \\
\hline O29 & $\mathrm{C} 7$ & C6 & & $100 \cdot 03(15)$ \\
\hline C32 & $\mathrm{C} 7$ & C6 & $1.517(3)$ & $114 \cdot 6(2)$ \\
\hline C9 & C8 & $\mathrm{C} 7$ & $1 \cdot 548(3)$ & $115 \cdot 3(2)$ \\
\hline $\mathrm{C} 10$ & C9 & C33 & $1.528(3)$ & $112.0(2)$ \\
\hline $\mathrm{C} 10$ & C9 & $\mathrm{C} 8$ & & $109 \cdot 5(2)$ \\
\hline C33 & C9 & $\mathrm{C} 8$ & $1.528(4)$ & $110.5(2)$ \\
\hline $\mathrm{C} 11$ & $\mathrm{ClO}$ & O34 & $1.514(3)$ & $109 \cdot 4(2)$ \\
\hline $\mathrm{Cll}$ & $\mathrm{Cl} 10$ & C9 & & $116 \cdot 2(2)$ \\
\hline $\mathrm{O} 34$ & $\mathrm{ClO}$ & C9 & $1.475(3)$ & $109 \cdot 3(2)$ \\
\hline $\mathrm{C} 12$ & $\mathrm{C} 11$ & C38 & $1 \cdot 518$ & $113.7(2)$ \\
\hline $\mathrm{C} 12$ & $\mathrm{Cl1}$ & $\mathrm{C} 10$ & & $108 \cdot 0(2)$ \\
\hline C38 & $\mathrm{Cll}$ & $\mathrm{C} 10$ & $1.525(4)$ & $111.5(2)$ \\
\hline $\mathrm{C} 13$ & $\mathrm{C} 12$ & O36 & $1.527(3)$ & $105 \cdot 7(2)$ \\
\hline $\mathrm{C} 13$ & $\mathrm{Cl} 2$ & $\mathrm{Cll}$ & & $117 \cdot 2(2)$ \\
\hline O36 & $\mathrm{C} 12$ & $\mathrm{Cll}$ & $1.468(2)$ & $108 \cdot 8(2)$ \\
\hline C14 & $\mathrm{Cl} 3$ & C39 & $1.531(4)$ & $110.5(2)$ \\
\hline $\mathrm{C} 14$ & $\mathrm{Cl} 3$ & $\mathrm{C} 12$ & & $111.7(2)$ \\
\hline C39 & $\mathrm{C} 13$ & $\mathrm{C} 12$ & $1.525(3)$ & $109 \cdot 7(2)$ \\
\hline $\mathrm{C} 15$ & $\mathrm{Cl} 4$ & $\mathrm{O} 40$ & $1.523(4)$ & $108.9(2)$ \\
\hline C15 & $\mathrm{Cl} 4$ & $\mathrm{C} 13$ & & $114.5(2)$ \\
\hline $\mathrm{O} 40$ & $\mathrm{C} 14$ & $\mathrm{C} 13$ & $1.446(3)$ & $106 \cdot 5(2)$ \\
\hline $\mathrm{C} 16$ & $\mathrm{Cls}$ & $\mathrm{C} 14$ & $1.496(5)$ & $115.5(3)$ \\
\hline C19 & 018 & $\mathrm{C} 4$ & $1 \cdot 323(3)$ & $116.8(2)$ \\
\hline $\mathrm{O} 20$ & $\mathrm{C} 19$ & N21 & $1 \cdot 195(3)$ & $122.0(2)$ \\
\hline $\mathrm{O} 20$ & $\mathrm{C} 19$ & 018 & & $128 \cdot 2(2)$ \\
\hline $\mathrm{N} 21$ & $\mathrm{Cl9}$ & 018 & $1.407(3)$ & $109 \cdot 8(2)$ \\
\hline $\mathrm{C} 22$ & $\mathrm{~N} 21$ & $\mathrm{C} 25$ & $1 \cdot 368(4)$ & $106 \cdot 7(2)$ \\
\hline $\mathrm{C} 22$ & $\mathrm{~N} 21$ & $\mathrm{C} 19$ & & $127.9(2)$ \\
\hline $\mathrm{C} 25$ & $\mathrm{~N} 21$ & $\mathrm{C} 19$ & $1 \cdot 386(3)$ & $125 \cdot 3(2)$ \\
\hline $\mathrm{N} 23$ & $\mathrm{C} 22$ & N21 & $1 \cdot 303(4)$ & $111.8(3)$ \\
\hline C24 & $\mathrm{N} 23$ & $\mathrm{C} 22$ & $1 \cdot 388(5)$ & $104 \cdot 7(3)$ \\
\hline $\mathrm{C} 25$ & $\mathrm{C} 24$ & $\mathrm{~N} 23$ & $1 \cdot 339(5)$ & $111.5(3)$ \\
\hline $\mathrm{N} 21$ & $\mathrm{C} 25$ & $\mathrm{C} 24$ & & $105 \cdot 3(3)$ \\
\hline $\mathrm{C} 28$ & $\mathrm{O} 27$ & C6 & $1.428(3)$ & $106 \cdot 1(2)$ \\
\hline 029 & $\mathrm{C} 28$ & C30 & $1.440(2)$ & $110 \cdot 3(2)$ \\
\hline O29 & $\mathrm{C} 28$ & C31 & & $109 \cdot 3(2)$ \\
\hline O29 & $\mathrm{C} 28$ & $\mathrm{O} 27$ & & $105 \cdot 5(2)$ \\
\hline C30 & C28 & C3I & $1.509(5)$ & $113.5(3)$ \\
\hline C30 & $\mathrm{C} 28$ & 027 & & $108 \cdot 3(2)$ \\
\hline C31 & C28 & $\mathrm{O} 27$ & $1 \cdot 518(4)$ & $109 \cdot 6(2)$ \\
\hline C7 & O29 & $\mathrm{C} 28$ & & $109 \cdot 7(2)$ \\
\hline C35 & O34 & $\mathrm{C} 10$ & $1.329(3)$ & $123.8(2)$ \\
\hline 036 & C35 & 037 & $1.335(3)$ & $120 \cdot 0(2)$ \\
\hline 036 & C35 & $\mathrm{O} 34$ & & $120 \cdot 2(2)$ \\
\hline 037 & $\mathrm{C} 35$ & $\mathrm{O} 34$ & $1.203(3)$ & $119.8(2)$ \\
\hline $\mathrm{C} 12$ & 036 & C35 & & $121 \cdot 3(2)$ \\
\hline C41 & O40 & $\mathrm{C} 14$ & $1.405(4)$ & $114.8(2)$ \\
\hline $\mathrm{O} 42$ & $\mathrm{C} 41$ & 040 & $1.405(4)$ & $112 \cdot 2(3)$ \\
\hline C43 & $\mathrm{O} 42$ & C41 & $1.425(4)$ & $113.5(2)$ \\
\hline C44 & $\mathrm{C} 43$ & O42 & $1.498(4)$ & $109 \cdot 2(2)$ \\
\hline C45 & $\mathrm{C} 44$ & C49 & $1.391(4)$ & $117 \cdot 7(2)$ \\
\hline C45 & C44 & $\mathrm{C} 43$ & & $121 \cdot 6(2)$ \\
\hline C49 & C44 & $\mathrm{C} 43$ & $1.390(4)$ & $120.7(2)$ \\
\hline C46 & $\mathrm{C} 45$ & $\mathrm{C} 44$ & $1 \cdot 386(4)$ & $120 \cdot 3(3)$ \\
\hline $\mathrm{C} 47$ & C46 & $\mathrm{C} 45$ & $1.377(5)$ & $120 \cdot 7(3)$ \\
\hline C48 & C47 & C46 & $1 \cdot 378(4)$ & $119.5(3)$ \\
\hline C49 & C48 & C47 & $1.372(4)$ & $119 \cdot 7(3)$ \\
\hline C44 & C49 & C48 & & $122.0(2)$ \\
\hline
\end{tabular}

isotropic thermal parameters. The atom positions in the block not being refined served to define the origin along the screw axis. The function $\sum w\left(\left|F_{o}\right|-\left|F_{c}\right|\right)^{2}$ was minimized, where $w=1 /\left[\sigma\left(F_{o}\right)\right]^{2}$ and $\sigma\left(F_{o}\right)=$ $\left(0.5 k I^{-1 / 2}\left\{[\sigma(I)]^{2}+(0.02 I)^{2}\right\}^{1 / 2}\right)$. The intensity, $I$, is given by $\left(I_{\text {peak }}-I_{\text {backgound }}\right) \times($ scan rate $) ; 0.02$ is a factor to downweight intense reflections and to account for instrument instability and $k$ is the correction due to Lp effects and decay. $\sigma(I)$ was estimated from counting statistics; $\sigma(I)=\left[\left(I_{\text {peak }}+I_{\text {background }}\right)^{1 / 2}\right.$ $\times$ (scan rate)]. The final $R=0.0301$ for 3141 reflections, with $w R=0.0328 \quad\left(R_{\mathrm{all}}=0.0357, \quad w R_{\mathrm{all}}=\right.$ 0.0344 ) and $S=1 \cdot 140$. The maximum $|\Delta / \sigma|<0 \cdot 1$ in the final refinement cycle and the minimum and maximum peaks in the final $\Delta F$ map were -0.19 and $0 \cdot 14 \mathrm{e} \AA^{-3}$, respectively. The scattering factors for the non- $\mathrm{H}$ atoms were taken from Cromer \& Mann (1968), with the anomalous-dispersion corrections taken from the work of Cromer \& Liberman (1970). The scattering factors for the $\mathrm{H}$ atoms were obtained from Stewart, Davidson \& Simpson (1965). Values used to calculate the linear absorption coefficient are from International Tables for X-ray Crystallography (1974, Vol. IV, p. 55).* Figures were generated using SHELXTL-Plus (Sheldrick, 1988). The positional and thermal parameters for non-H atoms are listed in Table 1, while the bond lengths and angles for the non- $\mathrm{H}$ atoms are listed in Table 2 . The atom-labeling scheme is shown in Fig. 1. Other computer programs used in this work are listed in reference 11 of Gadol \& Davis (1982).

Related literature. The asymmetric synthesis of the seco acid of erythronolide $\mathbf{B}$ has been recently

* Lists of anisotropic thermal parameters, $\mathrm{H}$-atom positional parameters, bond distances and angles involving $\mathrm{H}$ atoms, torsion angles, structure-factor amplitudes and a unit-cell packing diagram have been deposited with the British Library Document Supply Centre as Supplementary Publication No. SUP 53689 (37 pp.). Copies may be obtained through The Technical Editor, International Union of Crystallography, 5 Abbey Square, Chester CH1 2HU, England.

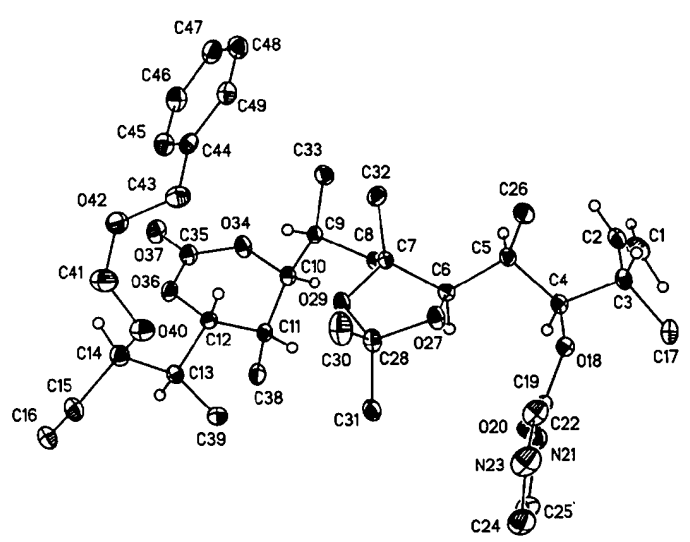

Fig. 1. View of (1) showing the atom-labeling scheme. Only the $\mathbf{H}$ atoms of the ethene moiety and those at chiral centers are shown. The non- $\mathrm{H}$ atoms are scaled to the $30 \%$ probability level while the $\mathrm{H}$ atoms are drawn to an arbitrary size. 
reported (Martin et al., 1989, and references therein). The crystal structure of a precursor of (1) and (2) has also been reported (Lynch, Pacofsky, Martin \& Davis, 1989).

Funding for this project was provided by the Robert A. Welch Foundation (F-652) and the National Institutes of Health (GM 31077) to SFM.

\section{References}

Cromer, D. T. \& Liberman, D. (1970). J. Chem. Phys. 53, 1891-1898.

Cromer, D. T. \& MANN, J. B. (1968). Acta Cryst. A24, 321-324.
Evans, D. A., Chapman, K. T. \& Carreira, E. M. (1988). J. Am. Chem. Soc. pp. 3560-3578.

Gadol, S. M. \& Davis, R. E. (1982). Organometallics, 1, 1607-1613.

Lynch, V. M., Pacofsky, G. J., Martin, S. F. \& Davis, B. E. (1989). Acta Cryst. C45, 973-975.

MARTIN, S. F. \& LeE, W.-C. (1990). In preparation.

Martin, S. F., Pacofsky, G. J., Gist, R. P. \& LeE, W.-C. (1989). J. Am. Chem. Soc. 111, 7634-7636.

SHELDRICK, G. M. (1976). SHELX76. Program for crystal structure determination. Univ. of Cambridge, England.

SHELDRICK, G. M. (1988). SHELXTL-Plus Structure Determination Software Programs. Nicolet XRD Corporation, Madison, Wisconsin, USA.

Stewart, R. F., Davidson, E. R. \& Simpson, W. T. (1965). $J$. Chem. Phys. 42, 3175-3187.

Acta Cryst. (1991). C47, 1120-1122

\title{
Structure of 6-(4-n-Propoxybenzoyloxy)flavone
}

\author{
By TaKahiro Kawai, Yukio Yoshimura and Kohji Shimaoka \\ Department of Physics, Faculty of Science and Engineering, Ritsumeikan University, Tojiin-kitamachi, \\ Kita-ku, Kyoto 603, Japan \\ NAOTAKE NAKaMURA \\ Department of Chemistry, Faculty of Science and Engineering, Ritsumeikan University, Tojiin-kitamachi, \\ Kita-ku, Kyoto 603, Japan \\ AND SHINICHI YANO \\ Department of Chemistry, Faculty of Engineering, Gifu University, Yanagido, Gifu 501-11, Japan
}

(Received 11 July 1990; accepted 7 November 1990)

\begin{abstract}
Oxo-2-phenyl-4H-1-benzopyran-6-yl 4propoxybenzoate, $\mathrm{C}_{25} \mathrm{H}_{20} \mathrm{O}_{5}, M_{r}=400 \cdot 43$, triclinic, $P \overline{1}, \quad a=16.306(11), \quad b=14.830(12), \quad c=$ $13.918(20) \AA, \quad \alpha=107.24(9), \quad \beta=99.80(9), \quad \gamma=$ $72.33(6)^{\circ}, \quad V=3050(5) \AA^{3}, \quad Z=6, \quad D_{x}=$ $1.306 \mathrm{~g} \mathrm{~cm}^{-3}, \quad \lambda($ Mo K $\alpha)=0.71073 \AA, \quad \mu=$ $0.849 \mathrm{~cm}^{-1}, \quad F(000)=1260, T=298 \mathrm{~K}, R=0.076$, $w R=0.047$ for 10683 unique observed reflections. There are no unusual bond distances or angles. The flavone skeleton is twisted with respect to the 4-npropoxybenzoyloxy group in the molecule.
\end{abstract}

Experimental. The title compound, 6-(4-n-propoxybenzoyloxy)flavone, was prepared by a conventional dehydrochloride reaction of 6-hydroxyflavone and 4-n-propoxybenzoyl chloride in a mixture of benzene and pyridine (Hirose, Tsuya, Nishigaki, Idaka \& Yano, 1989) and purified by recrystallization from acetone. A single crystal was obtained as a transparent and elongated rod by slow evaporation from acetone solution. A crystal with approximate dimensions $0.5 \times 0.5 \times 0.5 \mathrm{~mm}$ was mounted on a Rigaku
ROTA AFC-5 diffractometer equipped with a graphite monochromator. The lattice parameters were obtained from the setting angles of 25 reflections with $5<2 \theta<10^{\circ}$, Mo $K \alpha$ radiation. Intensities were measured within the ranges $0<2 \theta<65^{\circ},-20$ $\leq h \leq 20,-20 \leq k \leq 20,0 \leq l \leq 15$, using $\omega-2 \theta$ scans. Intensities of three standard reflections $(520$, $42 \overline{4}, 624)$ were measured every 100 reflections (maximum correction on $I$ was $<1.8 \%$ ). Lorentz and polarization corrections but no absorption correction were applied. 10683 unique observed reflections were measured with $F_{o} \geq 2 \sigma\left(F_{o}\right)$. The structure was determined by direct methods using RANTAN (Yao, 1981). The $\mathrm{H}$-atom positions were geometrically obtained, non- $\mathrm{H}$ atoms refined with anisotropic thermal parameters. Final refinement including $\mathrm{H}$ atoms was made by full-matrix leastsquares minimization using UNICS (Sakurai, 1967). Atomic scattering factors are from International Tables for X-ray Crystallography (1974, Vol. IV). The function $\sum w\left(F_{o}-F_{c}\right)^{2}$ was minimized, where $w=$ $1 / \sigma\left(F_{o}\right)^{2}$. Final $R=0.076, w R=0.047, \quad S=5.41$,

(C) 1991 International Union of Crystallography 